\title{
Modeling of heat transfer deterioration regimes when concentrating solutions in industrial film evaporators
}

\author{
Valentyn Petrenko, Yaroslav Zasyadko, Mykola Pryadko
}

\author{
National University of food Technologies, Kyiv, Ukraine
}

\section{Keywords:}

Evaporation

Film

Heat transfer

Concentration

\section{Article history:}

Received 14.11.2018

Received in revised

form 15.03.2019

Accepted 31.05.2019

\section{Corresponding \\ author:}

Yaroslav Zasyadko

E-mail:

Iaroslav@nuft.edu.ua
DOI: $10.24263 / 2304-$

974X-2019-8-2-12

\section{Abstract}

Introduction. Heat transfer deterioration in film evaporators may occur without breaking the integrity of film is termed as boiling crises of the 2-type (dry out). The mechanism and mathematical model of heat transfer at ultrahigh concentrations are developed.

Materials and methods. Physical modeling of the thermalhydrodynamic characteristics of the annular down flowing vapor-liquid streams of highly concentrated sugar solutions is carried out in an experimental set up with a pipe of $9 \mathrm{~m}$ long, $30 \mathrm{~mm}$ diameter, divided into 20 sections for discrete measurements of the heat flow, fluid concentration and film temperature of the pipe wall.

Results and Discussions. The mathematical modeling of heat transfer in flowing films of dense sugar solutions is based on the equations of convection and thermal conductivity with a parabolic velocity profile in the film. The model, shows that the correspondence with the experimental data on heat flux decrease along the evaporation channel occurs due to the periodic film mixing by the large waves (inflows). The mode of heat transfer deterioration is ascribed to the interaction of cyclical processes of increasing concentration on the interphase surface with the correspondent increase in temperature depression. Analytical expressions for calculating the increased interphase concentrations and decrease of the heat flux are provided within the wave cycles. The reduction of the wall heat flux along the entire channel is accounted for by means of zonal calculations with a step corresponding to the length of the large waves. Experiments do not show an abrupt decrease of heat flux along the pipe, but the reduction of HTC at concentrations of $75 \%$ and higher. The Prandtl numbers varied within the values of 25-350 along the evaporative pipe, accompanying the decrease in heat flux. The recommended correlations are valid within the heat flux variation of $3-15 \mathrm{~kW} / \mathrm{m}^{2}$, wetting flow rate $0,05-0,3 \mathrm{~kg} / \mathrm{m} \mathrm{s}$. Maximum deviation of the HTC calculated values from those observed in practice not more $15 \%$.

Conclusions. The developed model, depicting the process of heat transfer in film with a super-high concentration of liquids, adequately reflects the processes of heat transfer deterioration to dense films in long channels. 


\section{Introduction}

A number of research projects of the heat transfer to the falling liquid films point out that there might happen a critical deterioration of heat transfer which is attributed to the disruption of the liquid film integrity. This usually occurs as a result of film thinning due to the evaporation and respective reduction of liquid flow rate in the film or it may also be accompanied by the liquid droplets entrainment by the co-current steam flow [1]. Another mechanism of heat transfer deterioration which is caused by the non-linear interaction of gravity - capillary waves and oscillating thermocapillary instability has been addressed in [2]. This effect (called effect Marangoni), when applied to the liquid film flows, cases disruption of the film integrity and appearing of a system of rivulets, which in turn, causes a significant decrease in heat transfer along with the local overheating of heat transfer surface.

The mechanism of the film's integrity disruption due to the local film dry out is addressed in [2, 3] and the disintegration of films as a result of the Marangoni effect - in [4, 6]. In case of the significant increase in the concentration of evaporated solutions in the regimes of down flows in the industrial film evaporators, a noticeable growth of the concentrated solution's viscosity takes place. This leads to the lowering of the film's speed along with the respective increase of its thickness. This factor might be preventing in the disruption of the liquid film. Thus, when analyzing the process of a solution concentration, one may mark two competing effects: the first is - film's thinning down along the evaporative surface due to the evaporation of liquid, which leads to the film disruption; the other one-gradual increase of liquid's viscosity due to the solvent evaporation, which cases film thickening and thus, prevents loss of its integrity. In the event of the thick highly concentrated solutions evaporation, the rate of liquid viscosity growth prevails over the decrease in the flow rate due to the evaporation. Therefore in the above conditions, the mean film thickness will definitely grow up, in contrast to the similar processes of water films evaporation.

The objective of the present work is to develop a mechanism and is mathematical description of heat transfer deterioration in film evaporators may occur without breaking the integrity of film is termed as boiling crises of the 2-type (dry out).

\section{Materials and methods}

The experimentation has been carried out on the specially designed unit, which allowed modeling of real downward co-current steam - highly concentrated sugar solution films. The installation consisted of a $9 \mathrm{~m}$ height vertical pipe of stainless steel A304, internal diameter of $30 \mathrm{~mm}$, divided into 20 sections each $440 \mathrm{~mm}$ long.

The experimental pipe was encased into the outer pipe which served as a steam chamber for heating the inner experimental pipe. Maintaining constant steam temperature in the outer chamber provided a uniform along the pipe heating, and simultaneously modeled actual condition of heating in the industrial evaporators. The said sections were equipped with special pockets surrounding the lower part of each section with the drainage piping, allowing collecting condensate from the pipe sections and determining the distribution of heat flux along the pipe. The heating of the model tube was carried out with a dry saturated vapor. In the course of experiments, the temperature of the wall of the pipe was measured at 20 points, on each section by copper-constantan (type T) thermocouples.

Special probes positioned at each section allowed measurements of pressure head, concentration of solutions. In addition to the local data the following parameters were 
measured: initial solution and steam inlet flow rates, temperatures, solution concentration, heating steam temperature. Such system of measurements provided a possibility to double check the accuracy of measurements.

The current film flow rate was determined based on the local measured values of concentration and compared with that determined by means of heat balance based on the amount of condensate collected on the previous sections, which was equivalent to the amount of evaporated solvent.

The independent inlet flow parameters allowed formation of flows with fully developed wavy structures thus modeling real flows in the industrial evaporators with taking into account a variety of factors influencing heat transfer and hydrodynamics of film flows.

\section{Results and discussion}

\section{Concentration field modeling at constant film surface heat flux}

For high viscosity liquid films, the predominant mode of their motion is laminar, so during the evaporation of water (solvent) within the boundary of the interphase surface a thin layer with a concentration greater than that in the bulk of the film is being formed. Since the concentration equalization mechanism is extremely slow due to molecular diffusion only, in the absence of mixing, the concentration gradient can be significant and the boiling temperature on the film surface should be higher than the boiling temperature calculated at the average concentration. In dense films, the value of physic- chemical temperature depression is commensurable with the total temperature head, so the latter factor becomes determinant in the decrease of the heat flux along the channel during the concentration of dense solutions.

To find out the influence of the uneven distribution of the concentration on the heat exchange, let us consider an element of the plane film, of saturated solution with the initial concentration $C_{o}$ flowing along the vertical surface in the evaporation mode, Figure 1.

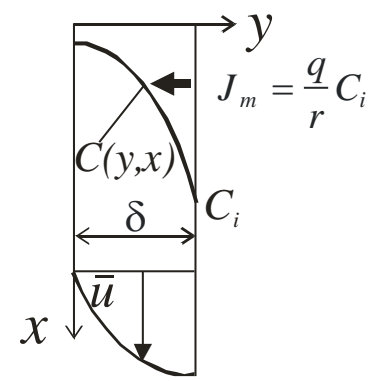

Figure 1. Schematics of the concentration distribution across the film at the evaporation from the interphase
In the process of concentration, the solvent evaporates, and the film thins at a rate $\frac{q}{r \rho}$. On the interfacial surface $(y=\delta)$ the concentration increases forming a profile $C_{i}=C(\delta, x)$, therefore, as the solvent disappears, a normal mass flow of the dissolved component is being formed $J_{m}=\frac{q}{r} C_{i}$, which diffuses into the bulk of film due to molecular or turbulent diffusion. The equilibrium condition of the flows of the dissolved component on the interphase surface when taking account of the molecular diffusion only, will be expressed as:

$$
-D_{m} \frac{\partial C(\delta, x)}{\partial y}=\frac{q}{r \rho} C(\delta, x),
$$


where $q$-heat flux, $r$-specific heat of evaporation, $\rho$ - liquid density, $y, x$ - normal and longitudinal to the heat surface coordinate, $\delta$ - film thickness, $D_{m}-$ molecular diffusion coefficient.

Equation (1) represents a boundary condition which holds on the interphase at $y=\delta$ and valid for the convection-diffusion equation

$$
u \frac{\partial C(x, y)}{\partial x}=D_{m} \frac{\partial^{2} C(x, y)}{\partial y^{2}}
$$

Since the transverse mass flux on the heated wall equals 0 , then:

$$
\frac{\partial C(0, x)}{\partial y}=0 \text {. }
$$

Assuming an uniform velocity and concentration distribution on the inlet at $x=0$

$$
u(y, 0)=\bar{u}, C(y, 0)=C_{o} .
$$

If, further on, we assume that the effect of local velocity upon the left side of (2) may be accounted only by its mean value $\bar{u}(x)$, then the analytical solution of $(1,2,3)$ will be [7]:

$$
C(x, y)-C_{o}=1-\sum_{n=1}^{\infty} \frac{2 \operatorname{Sin} \mu_{n}}{\mu_{n}+\operatorname{Sin} \mu_{n} \operatorname{Cos} \mu_{n}} \operatorname{Cos}\left(\mu_{n} \frac{y}{\delta}\right) \exp \left(-\mu_{n}^{2} \frac{D_{m} x}{\bar{u} \delta^{2}}\right) .
$$

The respective characteristic equation for finding eigenvalues will be:

$$
\operatorname{ctg} \mu_{n}=\frac{\mu_{n}}{P e_{m v}},
$$

where $-P e_{m v}=\frac{q \delta}{r \rho D_{m}}-$ mass transfer Peclet number, expressed through the evaporation velocity $\frac{q}{r \rho}$.

The development of the concentration profiles along the evaporation wall is presented in Figure 2. 


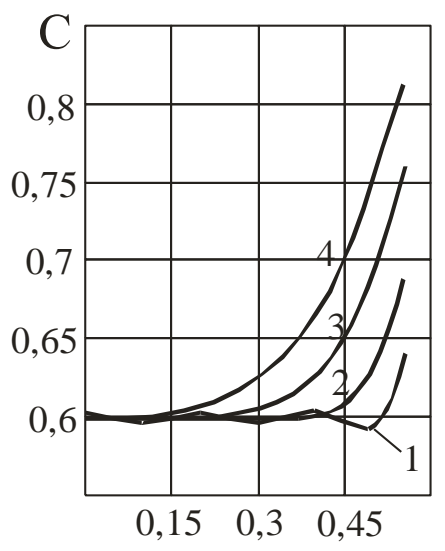

Figure 2. Concentration profiles in the downward film of concentrated sugar solution at a distance $x$ from the evaporation channel $1-x=0,1 \mathrm{~m} ; 2-0,8 ; 3-2,5 ; 4-5$, $P e_{m v}=1 ; \mathrm{CP}_{\mathrm{o}}=60 \% ; \mathrm{t}=80{ }^{\circ} \mathrm{C}$;

$\Gamma_{v}=0,15 \cdot 10^{3} \frac{\mathrm{m}^{2}}{\mathrm{~s}} ; v=3,92 \cdot 10^{-6} \frac{\mathrm{m}^{2}}{\mathrm{~s}} ;$ $\rho=1260 \frac{\mathrm{kg}}{\mathrm{m}^{3}} ; D=1 \cdot 10^{9} \frac{\mathrm{m}^{2}}{\mathrm{~s}} ; r=2308 \frac{\mathrm{kJ}}{\mathrm{kg}} ;$

$\delta, m m$

$$
\begin{aligned}
& q=5,14 \frac{k W}{m^{2}} ; \mu_{1}=0,8603 ; \mu_{2}=3,4256 ; \\
& \mu_{3}=6,4373 ; \mu_{4}=9,5293 ; \mu_{5}=12,6453 ; \\
& \mu_{6}=15,7713 .
\end{aligned}
$$

Interphase fluid concentration $C(x, \delta)$ may be found from (4) at $y=\delta$ :

$$
C(x, \delta)-C_{o}=1-\sum_{n=1}^{\infty} \frac{2 \operatorname{Sin} \mu_{n} \operatorname{Cos} \mu_{n}}{\mu_{n}+\operatorname{Sin} \mu_{n} \operatorname{Cos} \mu_{n}} \exp \left(-\mu_{n}^{2} \frac{D_{m} x}{\bar{u} \delta^{2}}\right) .
$$

\section{Modeling of concentration field at constant mass of dissolved component flux from film interface towards its bulk}

The equations (4 and 6) are inconvenient in usage, since at every certain value of the heat flux and film thickness one should determine respective values of eigenvalues $\mu_{n}$ by (5) at the conditions of constant heat flux.

In real multi effect evaporators the heating surface is being heated by the steam, and therefore due to the increase of the film concentration, there is a respective growth in local physico-chemical temperature depression. This leads to the decrease in the positive temperature head, which eventually causes a gradual decrease, proportional to the positive temperature difference, of the local heat flux along the pipe. In the said conditions, it looks much more advisable to formulate the boundary conditions (1) in terms of $q C_{i}$ constancy instead of constant $q$. This is equivalent to the constancy of the transversal mass flux of dissolve component $J_{m}=\frac{q}{r} C_{i}$, which holds true in a certain range of solution concentrations and temperature differences. Thus, (1) can be rearranged as follows:

$$
-D_{m} \frac{\partial C(\delta, x)}{\partial y}=\frac{q}{r \rho} C(\delta, x) \approx \frac{J_{m}}{\rho}=\text { const } .
$$

With the precise analytical solution of (2) with the boundary conditions $(3,7)$ [7]: 


$$
C(\eta, \xi)=C_{o}+\frac{2 J_{m}}{\rho D_{m}} \sqrt{\frac{D_{m} x}{\bar{u}}}\left\{\frac{1}{\sqrt{\pi}} \exp \left(-\frac{(\delta-y)^{2} \bar{u}}{4 D_{m} x}\right)-\frac{(\delta-y)}{2 \sqrt{D_{m} \frac{x}{\bar{u}}}} \operatorname{erfc}\left(\frac{(\delta-y)}{2 \sqrt{D_{m} \frac{x}{\bar{u}}}}\right)\right\}
$$

]The results of calculations of (4) and (8) are given in Figure 3.
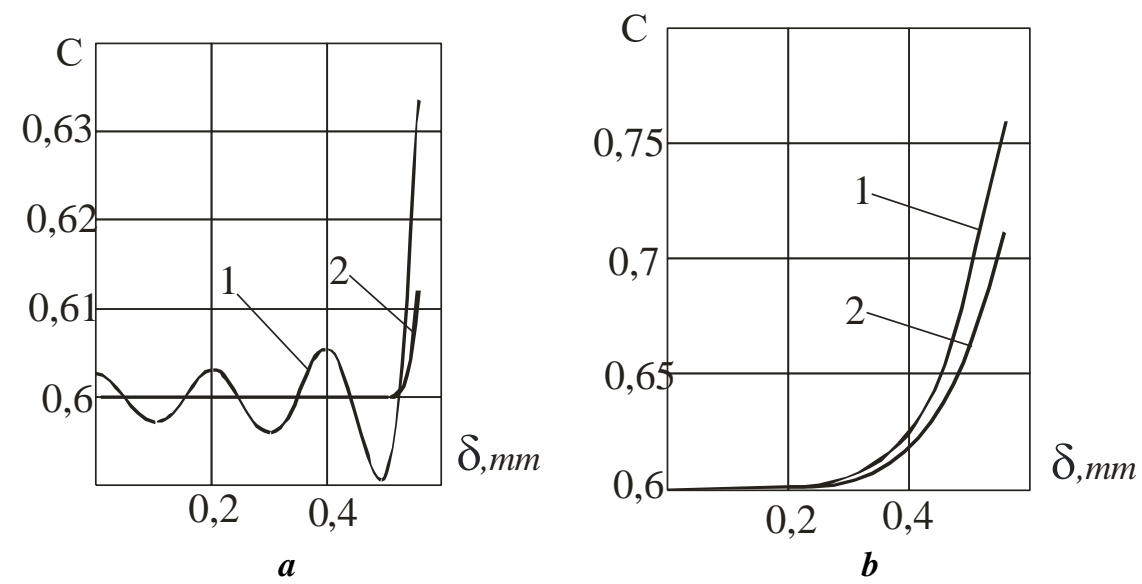

Figure 3. Calculated profiles of concentration across the film at the initial concentration of 60\% DM:

$$
\begin{gathered}
\Gamma_{v}=0,15 \cdot 10^{3} \frac{\mathrm{m}^{2}}{\mathrm{~s}} ; v=3,92 \cdot 10^{-6} \frac{\mathrm{m}^{2}}{\mathrm{~s}} ; \rho=1260 \frac{\mathrm{kg}}{\mathrm{m}^{3}} ; D_{m}=1 \cdot 10^{9} \frac{\mathrm{m}^{2}}{\mathrm{~s}} ; \\
r=2308 \frac{\mathrm{kJ}}{\mathrm{kg}} ; q=5,14 \frac{\mathrm{kW}}{\mathrm{m}^{2}} . \\
\text { a: } x=0,05 \mathrm{~m} ; 1-\text { calculated by }(4), 2-\text { by }(8) ; \\
\text { b: } x=2,5 \mathrm{~m} ; 1-\text { calculated by }(4), 2-\text { by }(8) .
\end{gathered}
$$

In the dimensionless form (8) can be written as:

$$
C(\eta, \xi)=C_{o}+\frac{2 J_{m} \delta}{\rho D_{m}} \sqrt{\frac{4 \xi}{P e_{m}}}\left\{\frac{1}{\sqrt{\pi}} \exp \left(-\frac{(1-\eta)^{2}}{\frac{16 \xi}{P e_{m}}}\right)-\frac{(1-\eta)}{2 \sqrt{\frac{4 \xi}{P e_{m}}}} \operatorname{erfc}\left(\frac{(1-\eta)}{2 \sqrt{\frac{4 \xi}{P e_{m}}}}\right)\right\},
$$

where $P e_{m}=\frac{4 \Gamma_{v}}{D_{m}}-$ diffusion Peclet number, $\Gamma_{v}-$ volumetric liquid flux.

Fluid concentration on the interphace $C_{i}(\xi)$ can be found by (9) at $\eta=1$.

$$
C_{i}(\xi)=C_{o}+\frac{4 J_{m} \delta}{\rho D_{m} \sqrt{\pi}} \sqrt{\frac{\xi}{P e_{m}}}
$$




\section{Modeling of critical heat transfer deterioration to films with developed wave structure at evaporation from film surface}

The interphase surface of a dense viscous laminar film flowing down along a high vertical pipe is nearly smooth. Three-dimensional sickle-shaped swells periodically roll over the surface [8]. Under these conditions, the process of heat transfer is affected by the periodic perturbation of the film by wave-like rolls. These rolling swells mix the film and thus reduce the concentration on the interphase from its maximum value $C_{i}(\xi)$ to the mean in the bulk. Accordingly, the temperature distribution across the film also experiences periodic variations from the its initial profile, formed during the mixing process during the passage of the wave propagation, to the final, which is being formed at the moment of the next rolling swell approach. Given the fact that for low flow rates of the solution in the film and high viscosity of fluid, the film velocity profile after the perturbation initiated by the wave passage, transfers from the linear one to parabolic within a short section of a pipe. The heat transfer equation which determines the temperature field will be written:

$$
\left[\left(\frac{\tau_{i}}{v \rho \delta}+\frac{g}{v}\right) \eta-\frac{g}{2 v} \eta^{2}\right] \delta^{3} \frac{\partial \theta(\eta, \xi)}{\partial \xi}=a \frac{\partial^{2} \theta(\eta, \xi)}{\partial \eta^{2}}
$$

where $\eta=\frac{y}{\delta}-$ dimensionless transverse coordinates; $\xi=\frac{x}{\delta}-$ dimensionless longitudinal coordinates; $\tau_{i}$ - shear stress on the film surface; $v$ - cinematic viscosity coefficient, $a-$ temperature conductivity, $g$-acceleration of gravity; $\theta(\eta, \xi)=\frac{t(\eta, \xi)-t_{i}(\xi)}{t_{w}-t_{i}(\xi)}-$ dimensionless temperature; $t_{w}-$ wall temperature;

Approximate solution of (11) with the corresponding boundary conditions $\eta=0$, $\theta=1 ; \eta=1, \theta=0$, with the initial temperature distribution which is formed at the passage of a large wave at $\xi=\xi_{m}$ and is given as:

$$
\theta\left(\eta, \xi_{m}\right)=\left(\frac{P e}{4}\right) \frac{1}{D} \exp \left(-\frac{\xi_{m}}{D}\right)\left(\frac{\eta^{2}}{2}-\eta\right)+1
$$

is as follows [11]:

$$
\theta(\eta, \xi)=\frac{P e}{8}\left[\frac{R_{1}-1}{R}+\frac{P e}{80 D R} \frac{\left(16 \rho g \delta+25 \tau_{i}\right)}{\left(3 \tau_{i}+2 \rho g \delta\right)} \exp \left(-\frac{\xi_{m}}{D}\right)\right] \exp \left[\frac{\xi_{m}-\xi}{R}\right]\left(\eta^{2}-\eta\right)-\eta+1
$$

where $D=\frac{P e}{80} \frac{25 \tau_{i}+16 \rho g \delta}{3 \tau_{i}+2 \rho g \delta} ; R=\frac{\left(7 \rho g \delta+10 \tau_{i}\right)}{\left(3 \tau_{i}+2 \rho g \delta\right)} \frac{P e}{160} ; R_{o}=\frac{4 \tau_{i}+3 \rho g \delta}{7 \rho g \delta+10 \tau_{i}} \frac{40}{P e}$ 
$R_{1}=R R_{o}=\frac{\tau_{i}+\frac{3}{4} \rho g \delta}{3 \tau_{i}+2 \rho g \delta} ; P e=\frac{4 \Gamma_{v}}{a} ; \xi_{m}=D \ln \left(\frac{P e}{8 D}\right)-$ distance at which $\theta\left(1, \xi_{m}\right)=0$.

Under the conditions of tail film evaporators of the multi effect evaporation plants during the concentration of thick sugar syrups the characteristic values of positive temperature difference between the heating surface and film are being kept low. Therefore the heat flux and the co-current steam velocity are also low, even in case of long evaporative pipes. As a result, the shear stress on the interphase at the given conditions has little effect on the hydrodynamic parameters of the film. Under this condition, the movement of the film approximates the free downward flow on the vertical surface. In the case of free downward flow ( $\tau_{i}=0$ ), the above complexes $D, R, R_{o}, R_{1}$ can be written if the form:

$$
D=\frac{P e}{10}, R=\frac{7 P e}{320}, R_{o}=\frac{120}{7 P e}, R_{1}=R R_{o}=\frac{3}{8} .
$$

The temperature profile (13) transforms into :

$$
\theta(\eta, \xi)=\left[\frac{40}{7} \exp \left(-\frac{10}{P e} \xi_{m}\right)-\frac{25}{7}\right] \exp \left(\frac{320}{7 P e}\left(\xi_{m}-\xi\right)\right)\left(\eta^{2}-\eta\right)-\eta+1 .
$$

The dimensionless coordinate $\xi_{m}$ at which $\theta\left(1, \xi_{m}\right)=0$, can be found from the following:

$$
\xi_{m}=0,0223 P e
$$

During evaporation, the temperature on the film surface equals to that of the steam plus the value of physical-chemical depression, which is determined by the local dry matter concentration:

$$
t_{i}(\xi)=t_{\text {sat }}+R_{\text {dep }}(\xi)
$$

The relationship between the value of physical-chemical depression and local solution concentration $R_{\text {dep }}(\xi)$ may be expressed as a piecewise two-section approximation within the concentration intervals $0,37<C<0,75$ as:

$$
R_{\text {dep }}(\xi)=16,22 \frac{T^{2}}{r}\left(\frac{C_{i}(\xi)}{a-b C_{i}(\xi)}\right) .
$$

And $0,75<\mathrm{C}<0,9$ as:

$$
R_{d e p}(\xi)=\left(1156,9-6322 C_{i}(\xi)+1,3 \cdot 10^{4} C_{i}^{2}(\xi)-11920 C_{i}^{3}(\xi)+4134 C_{i}^{4}(\xi)\right) 16,22 \frac{T^{2}}{r}
$$

where $a=0,62655, \sigma=0,695$.

The temperature distribution within the film may be denoted as a function of the interphase temperature $t_{i}(\xi)$ : 


$$
t(\eta, \xi)=t_{i}(\xi)+\left[t_{w}-t_{i}(\xi)\right] \theta(\eta, \xi)
$$

or, making use of (16), it may be expressed through the saturated steam temperature $t_{\text {sat }}$ :

$$
t(\eta, \xi)=t_{s a t}+\left(t_{w}-t_{s a t}\right) \theta(\eta, \xi)+R_{d e p}(\xi)(1-\theta(\eta, \xi))
$$

The value of heat flux on the wall $q_{w}(\xi)$ can be found using $(14,20)$ keeping in mind that

which leads to:

$$
q_{w}(\xi)=-\left.\frac{\lambda}{\delta} \frac{d t(\eta, \xi)}{d \eta}\right|_{\eta=0}
$$

$$
q_{w}(\xi)=\frac{\lambda}{\delta}\left\langle\left[\frac{40}{7} \exp \left(-\frac{10 \xi_{m}}{P e}\right)-\frac{25}{7}\right] \exp \left(\frac{320}{7 P e}\left(\xi_{m}-\xi\right)\right)+1\right\rangle\left[t_{w}-t_{\text {sat }}-R_{d e p}(\xi)\right]
$$

where $\lambda$ - liquid thermal conductivity.

Since $\xi$ is measured from $\xi_{m}$, the expression for $R_{d e p}(\xi)$ within the concentration range less than $75 \%$ ( $C \leq 0,75)$ will acquire the form:

$$
R_{d e p}(\xi)=\frac{C_{o}+\frac{4 J_{m} \delta}{\rho D \sqrt{\pi}} \sqrt{\frac{\xi-\xi_{m}}{P e_{m}}}}{a-b\left(C_{o}+\frac{4 J_{m} \delta}{\rho D \sqrt{\pi}} \sqrt{\frac{\xi-\xi_{m}}{P e_{m}}}\right)} 16,22 \frac{T^{2}}{r}
$$

And within a range of $0,75<C<0,9 \quad-$

$$
R_{d e p}(\xi)=\left(\begin{array}{l}
1156,9-6322 C_{i}\left(\xi-\xi_{m}\right)+1,3 \cdot 10^{4} C_{i}^{2}\left(\xi-\xi_{m}\right)-11920 C_{i}^{3}\left(\xi-\xi_{m}\right)+ \\
+4134 C_{i}^{4}\left(\xi-\xi_{m}\right)
\end{array}\right) 16,22 \frac{T^{2}}{r}
$$

As it follows from, in case of a significant shear stress on the interphse as a result of a stem velocity over the liquid film, the suppression of physical-chemical depression which is accounted for by the multiplier $H_{\text {dep }}$ to the $R_{\text {dep }}(\xi)(22,23)$

$$
H_{\text {dep }}=\exp \left(-1,07 \cdot 10^{-2} \sqrt{W e} \sqrt[3]{P e}\right)
$$

where $W e=\frac{\rho_{2} u^{2} d}{\sigma}-$ the Weber number; $u$-steam velocity; $\sigma$-surface tension, $\rho_{2}$ - steam density; $d$ - pipe diameter. 
Having taken into account the effects of physical-chemical depression, its suppression by the co-current steam flow, expressed by $(16,22,23,24)$, the positive available temperature difference will determine the value of the local wall heat flux, which under above assumptions can be written as:

$$
q_{w}(\xi)=\frac{\lambda}{\delta}\left\langle\left[\frac{40}{7} \exp \left(-\frac{10 \xi_{m}}{P e}\right)-\frac{25}{7}\right] \exp \left(\frac{320}{7 P e}\left(\xi_{m}-\xi\right)\right)+1\right\rangle\left[t_{w}-t_{\text {sat }}-R_{\text {dep }}(\xi) H_{\text {dep }}\right] .
$$

Figure 4 depicts the calculated values of the cyclic, respective to the passages of successive large waves, changes of wall heat flux along a high vertical pipe ( $9 \mathrm{~m} \mathrm{long}$ ) on a section of $1 \mathrm{~m}$ long (between $4^{\text {th }}$ and $5^{\text {th }} \mathrm{m}$ ) to the highly concentrated sugar solution film at a regime of evaporation from free surface.

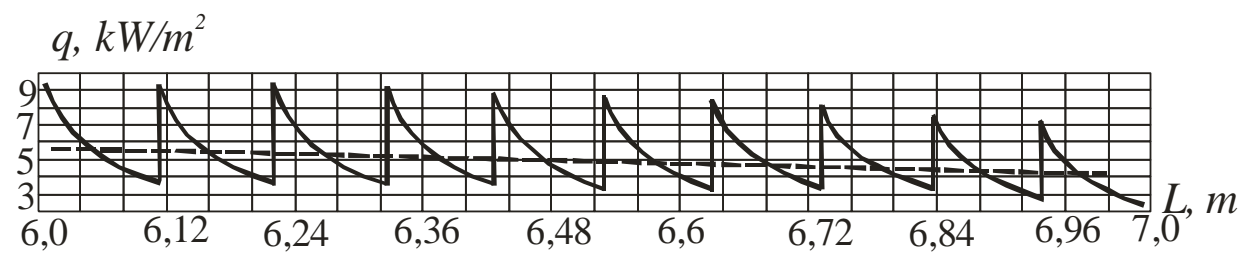

Figure 4. Profile of local values of wall heat flux along a high vertical pipe on the $1 \mathrm{~m}$ section (between the $4^{\text {th }}$ and $5^{\text {th }} \mathrm{m}$ ) to the highly concentrated sugar solufion flowing down in the regime of evaporation from the free surface.

Inlet fluid parameters: $D M_{n}=75,5 \% ; \Gamma_{v}=0,06110^{-3} \mathrm{~m}^{2}, / \mathrm{s}$;

$$
u_{2}-3,8 \mathrm{~m} / \mathrm{s}, t_{w}=115^{\circ} \mathrm{C}, t_{s a t}=100{ }^{\circ} \mathrm{C} . \lambda_{b w}=0,12 \mathrm{~m}
$$

The averaging of the heat flux values within the successive large waves has been carried out within the interval $\xi_{v}-\xi_{m}$ for each wave cycle :

$$
\bar{q}_{w}=\frac{1}{\xi_{v}-\xi_{m}} \int_{\xi_{m}}^{\xi_{v}} q_{w}(\xi) d \xi .
$$

In (26) the parameter $\xi_{m}$ has been calculated as per (15), whereas $-\xi_{v}=\xi_{m}+\frac{\lambda_{b w}}{\delta}$, where $\lambda_{b w}-$ the length of big waves and $\delta=\sqrt[3]{\frac{3 \Gamma_{v} v}{g}}-$ represents the calculated film thickness. The length of large waves for the free down flow of cold water films in 25-32 mm diameter pipes ranged from 0.1 to $0,14 \mathrm{~m}$. A similar result has been found at free film flow of concentrated apple syrup on a vertical flat surface.Making use of the above, when calculating the heat flux distribution along the pipe, the interval $\xi_{v}-\xi_{m}$ is calculated based upon the condition of the large waves length $\lambda_{b w}=0,12 \mathrm{~m}$. The character of the heat flux distribution is practically solely determined by the distribution of the useful temperature head as a function of the physical - chemical temperature depression value, which, in turn, is a function of concentration of the solution on the interphase boundary, Figure 5. 


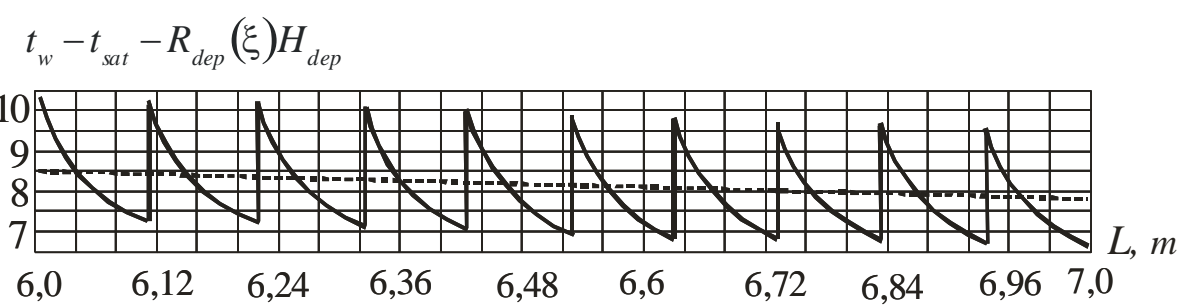

Figure 5 . Temperature head distribution along the $9 \mathrm{~m}$ long pipe on the section of $1 \mathrm{~m}$ long (between $4^{\text {th }}$ and $5^{\text {th }} \mathrm{m}$ ) at the regime of evaporation from the interphase surface. Inlet parameters - are the same as in Figure 4.

It is quite clear that due to the evaporation, there will be a certain film concentration growth along the flow as well as across the film. Moreover, there will be a noticeable concentration growth within a length of a single inter wave cycle, which is shown in Figure 6.

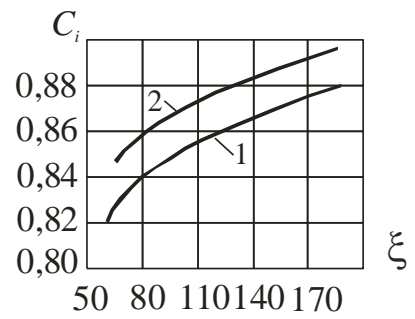

Figure 6. Film free surface concentration distribution $C_{i}$ within a single wave cycle. Data presented in Figure 6, are obtained for the flow in $9 \mathrm{~m}$ high pipe, curve $1-$ at a $4 \mathrm{~m}$ distance from the pipe inlet, relates to the $1^{\text {st }}$ cycle as per data in Figure 4,5. Curve $2-5 \mathrm{~m}$ distance, relates the $10^{\text {th }}$ cycle.
The calculation results of the film mean thickness, liquid viscosity and mean solution concentration within the pipe section from $4 \mathrm{~m}$ to $9 \mathrm{~m}$ are presented in Figure 7, a, b, c.

The comparison of the experimental and calculation data related to the concentration of sugar solutions in the vertical pipes within the section between $4 \mathrm{~m}$ to $9 \mathrm{~m}$ is presented in Figure 8.
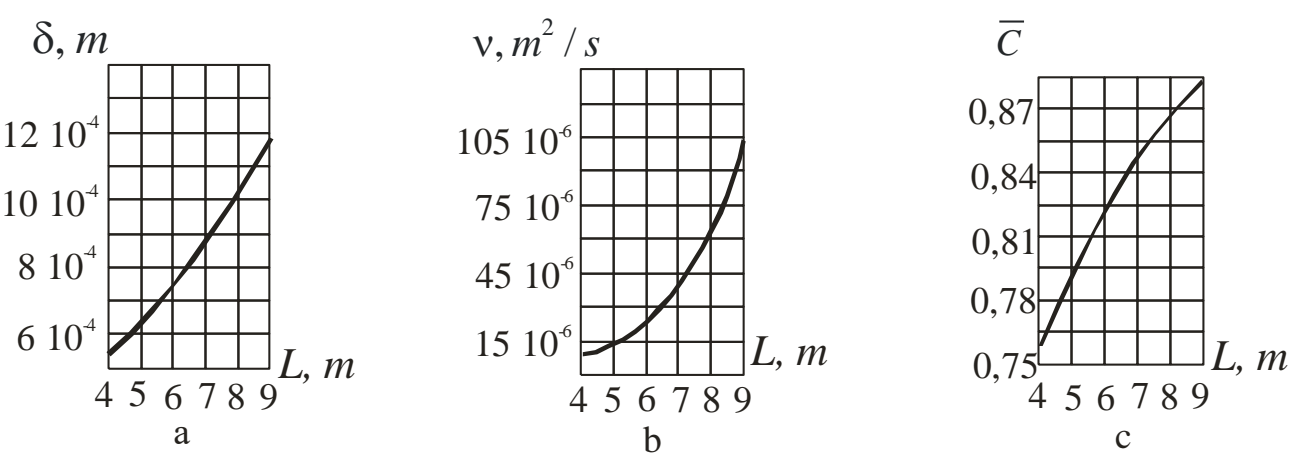

Figure 7. Mean values of the film thickness (a), viscosity (b) and solution concentration (c) within the pipe section from $4 \mathrm{~m}$ to $9 \mathrm{~m}$.

Inlet parameters - are the same as in Figure 4,5. 


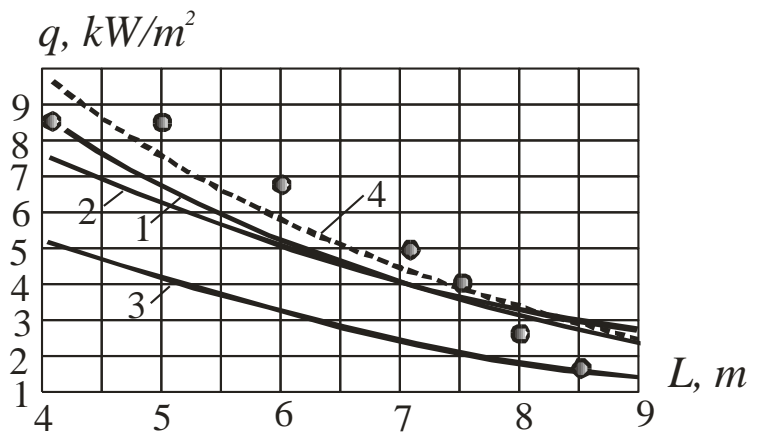

Figure 8. Comparison of the calculated and experimental data. Initial parameters of the solution are:

Sugar solution concentration $75,5 \% \mathrm{DM}$,

$\Gamma_{\mathrm{v}}=0,06110^{-3} \mathrm{~m}^{2} / \mathrm{s}, u=3,8 \mathrm{~m} / \mathrm{s}, \mathrm{t}_{\mathrm{w}}=115^{\circ} \mathrm{C}, \mathrm{t}_{\mathrm{sat}}=100{ }^{\circ} \mathrm{C}, \quad \lambda_{b w}=0,12 \mathrm{~m}$.

As it may be seen from Figure 8, the line 1 calculated as per (23-26) shows the same trend as the experimental data do, but deviates significantly form the experimental data, especially on the initial sections of the pipe where the error reaches $25 \%$ (within $4^{\text {th }}-5^{\text {th }} \mathrm{m}$ ).

Line 2 - is calculated based upon taking into account the mean over the section concentration $\bar{C}$ with the further calculation of physical-chemical depression and its reduction effect upon the heat transfer:

$$
q=\frac{\lambda}{\delta}\left(t_{w}-t_{s a t}-R_{d e p}\left(\bar{C}_{n i}\right)\right)
$$

where $R_{d e p}\left(\bar{C}_{n i}\right)=\left(1156,9-6322 \bar{C}_{n i}+1,3 \cdot 10^{4} \bar{C}_{n i}{ }^{2}-11920 \bar{C}_{n i}{ }^{3}+4134 \bar{C}_{n i}{ }^{4}\right) 16,22 \frac{T^{2}}{r}, \bar{C}_{n}-$ mean at every section fluid concentration,

$$
\bar{C}_{n}=\frac{\bar{C}_{n-1} \Gamma_{v}^{n-1}}{\Gamma_{v}^{n-1}-\frac{\bar{q}_{n} \delta_{n}\left(\xi_{v}-\xi_{m}\right)}{\rho r}},
$$

$\bar{C}_{n-1}$ - mean fluid concentration at the section inlet, $\Gamma_{v}^{n-1}$ - mass flow wetted perimeter, $\bar{q}_{n}-$ mean heat flux on a section (26). As it follows from Figure 8 , this methodology does not lead to more close correlation of the data. The deviation on the initial pipe sections grows even bigger as compared to the line 1 .

Line (3) - is calculated based on the mean interphase fluid concentration $\bar{C}_{n i}$.

$$
q=\frac{\lambda}{\delta}\left(t_{w}-t_{s a t}-R_{d e p}\left(\bar{C}_{n i}\right)\right)
$$

where $R_{d e p}\left(\bar{C}_{n i}\right)=\left(1156,9-6322 \bar{C}_{n i}+1,3 \cdot 10^{4} \bar{C}_{n i}{ }^{2}-11920 \bar{C}_{n i}{ }^{3}+4134 \bar{C}_{n i}{ }^{4}\right) 16,22 \frac{T^{2}}{r}$,

$$
\bar{C}_{n i}=\frac{1}{\xi_{v}-\xi_{m}} \int_{\xi_{m}}^{\xi_{v}} C_{i}(\xi) d \xi=\bar{C}_{n-1}+\frac{8 P e_{m} J_{m} \delta}{3 \sqrt{\pi} \rho D_{m}\left(\xi_{v}-\xi_{m}\right)}\left(\sqrt{\left(\frac{\xi_{v}}{P e_{m}}\right)^{3}}-\sqrt{\left(\frac{\xi_{m}}{P e_{m}}\right)^{3}}\right) .
$$


Despite of its greatest complexity, this calculation method gives the worst congruency with the experimental data. This may be explained by not taking into consideration the effect of physical-chemical depression reduction can be calculated by (24).

Line (4) - is calculated by the interval approximation method with further taking in account the reduction effect of co-current steam flow by the equations (28) taken as per mean fluid concentrations at sections $\bar{C}_{n}(27)$

$$
\begin{gathered}
q=\alpha\left(t_{w}-t_{\text {sat }}-H_{\text {dep }} R_{\text {dep }}\left(\bar{C}_{n}\right)\right) \\
\frac{\alpha}{\lambda}\left(\frac{v^{2}}{g}\right)^{1 / 3}=1,12 \operatorname{Re}^{-1 / 3}\left(0,85+0,01 P e^{0,2}+4,5 \cdot 10^{-4} P e^{0,86} \operatorname{Pr}^{-0,2}\right) K_{w} K_{b o i l} K_{L d}
\end{gathered}
$$

where $K_{L d}=\left\{1+0,06\left(\frac{v}{v_{o}+v}\right)\left[1-\exp \left(-0,05 L^{3}\right)\right]\right\}\left(\frac{d}{d_{o}}\right)^{0,35-0,06\left(\frac{d}{d_{o}}\right)}$;

$K_{w}=\sqrt{1+\left[7,5 \cdot 10^{-6} \operatorname{Re}_{2}\left(\rho / \rho_{2}\right)^{0,2}\right]^{2}}, K_{b o i l}=1 ; \operatorname{Re}=\frac{4 \Gamma_{v}}{v} ; \operatorname{Re}_{2}=\frac{u d \rho_{2}}{\mu_{2}} ;$

$\mu_{2}$ - steam viscosity dynamic coefficient, $\mathrm{Pa} \cdot \mathrm{s}$;

$v_{o}$ - water viscosity kinematic coefficient $100{ }^{\circ} \mathrm{C} ; L$ - pipe length, $m$;

$\alpha$ - the heat transfer coefficient (HTC), $\frac{W}{m^{2} s} ; d_{o}-0,02 m$.

As one may see, the proposed method renders the best congruity of the calculated and experimental data.

\section{Conclusions}

1. The proposed model of heat transfer in the liquid films as a process of periodic destruction of the surface layer of the super-high concentration solutions by large waves, which at high viscosity have the form of inflows, adequately reflects the processes of heat transfer deterioration to dense films in long channels in the mode of evaporation from the interphase surface.

2. The recommended calculation correlations and methodology allow obtaining data closely conforming to those found experimentally and thus may be recommended to be used in the engineering calculations of the industrial film evaporators.

3. The main reason for the deterioration of heat transfer to dense films in the process of concentration is the excess of the value of physical-chemical temperature depression on the interphase boundary over the mean temperature head value due to the uneven distribution of concentration. In a lesser degree, the intensity of the heat transfer is influenced by the growth of the film thickness and the drop in the dense solution thermal conductivity as the fluid concentration increases.

4. Recirculation of a dense solution in film evaporators on the one hand leads to a deterioration of heat transfer due to the growth of the average length of the concentration pipe, and on the other - it promotes the activation of the wave process and disruption of a highly concentrated layer on the interphase surface, which causes the intensification of heat transfer. 


\section{References}

1. Oron A., Davis S.H. Bankoff S.G. (1997), Long-Scale Evolution of Thin Liquid Films, Reviews of Modem Physics, 69, 931, DOI:10.1103/RevModPhys.69.931

2. Joo S.W., Davis S.H., Bankoff S.G. (1996), A mechanism for rivulet formation in heated falling films, J. Fluid Mech., 321, pp. 279-298,

DOI: $10.1017 /$ S0022112096007720

3. Aktershev S.P., Alekseenko S.V. (2019), Thermocapillary instability and rivulet structure formation in uniformly heatedfalling liquid film, International Journal of Multiphase Flow, 114, pp. 115-127,

DOI: 10.1016/j.ijmultiphaseflow.2019.02.007

4. Zayzhev D.V. (2003), Termokapilyrniy razriv stekauzhey plenki zidkosti, Novosibirsk.

5. Bohn M.S., Davis S.H. (1993), Thermocapillary breakdown of falling liquid films at high Reynolds number, Int. Heat and Mass Transfer, 36, pp. 1875-1881, DOI: 10.1016/S0017-9310(05)80175-9

6. Wang M., Zhao J.,Duan R. (2019), Rivulet formulation in the flow of film down a uniformly heated vertical substrate, Engineering Applications of Computational Fluid Mechanics, 13(1), pp. 396-416, DOI: 10.1080/19942060.2019.1600028.

7. Bejian A., Kraus A.D. (2003), Heat Transfer Hendbook, J. Wiley \& Sons, Hoboken, New Jersey.

8. Pryadko M.O., Globa O.V., Forsyuk, A.V., Globa V.Z. (2015), Film flows in tubes of apple juice evaporators, Scientific Works of National University of Food Technologies, 21(2).

9. Tetiana Vasylenko, Sergii Vasylenko, Jeanna Sidneva, Vitalii Shutiuk (2014), Best available technology - innovative methodological framework efficiency of sugar production, Ukrainian Food Journal, 3(1), pp. 122-133.

10. Piotr Cyklis (2017), Industrial scale engineering estimation of the heat transfer in falling film juice evaporators, Applied Thermal Engineering, 123, pp. 1365-1373, DOI: 10.1016/j.applthermaleng.2017.05.194

11. Valentyn Petrenko, Yaroslav Zasyadko (2016), Heat Transfer Modeling in Downflowing Films, LAP LAMBERT Academic Publishing, Germany. 